Relations industrielles

Industrial Relations

\title{
The Japanese Factory: Aspects of Its Social Organization. By
} James C. Abegglen. Glencoe, Ill.: The Free Press, 1958. 155 pp. $\$ 3.50$.

\section{Roger Chartier}

Volume 15, numéro 4, octobre 1960

URI : https://id.erudit.org/iderudit/1021955ar

DOI : https://doi.org/10.7202/1021955ar

Aller au sommaire du numéro

Éditeur(s)

Département des relations industrielles de l’Université Laval

ISSN

0034-379X (imprimé)

1703-8138 (numérique)

Découvrir la revue

Citer ce compte rendu

Chartier, R. (1960). Compte rendu de [The Japanese Factory: Aspects of Its Social Organization. By James C. Abegglen. Glencoe, Ill.: The Free Press, 1958. 155 pp. \$3.50.] Relations industrielles / Industrial Relations, 15(4), 517-518.

https://doi.org/10.7202/1021955ar

Tous droits réservés (C Département des relations industrielles de l’Université Laval, 1960
Ce document est protégé par la loi sur le droit d'auteur. L'utilisation des services d'Érudit (y compris la reproduction) est assujettie à sa politique d'utilisation que vous pouvez consulter en ligne.

https://apropos.erudit.org/fr/usagers/politique-dutilisation/ 
Le premier chapitre est consacré aux normes internationales existantes et le dernier contient les conclusions générales qui se dégagent de l'étude. Le corps de louvrage, divisé en huit chapitres, examine la législation et la pratique en vigueur et en particulier les questions suivantes: sources des fonds et des biens syndicaux; fins auxquelles ces fonds et biens peuvent être affectés; administration interne; mesures volontaires permettant ou encourageant une gestion satisfaisante; surveillance de l'administration financière des syndicats pour les pouvoirs publics; sanctions applicables en cas d'utilisation illicite des fonds syndicaux et en cas de non-observation des prescriptions légales; dévolution des biens syndicaux en cas de liquidation ou de dissolution des syndicats

Deux annexes complètent l'étude. Le premier comprend les références aux lois et règlements cités; l'autre fournit une liste des principaux ouvrages à consulter.

Au cours de leur enquête, les rapporteurs ont observé que l'on peut constater une différence assez nette entre les deux grands groupes dans lesquels se classent les mouvements syndicaux du monde, différence qui s'explique par le degré de développement de leur pays respectif, indépendamment de toute considération idéologique. D'une part, lorsque le syndicalisme a atteint un degré de maturité, «même si les dispositions législatives sont différentes, les syndicats ont prouvé qu'ils avaient l'expérience nécessaire pour élaborer à leur gré des règles adéquates pour l'administration de leurs ressources et pour en assurer effectivement le respect ». Par ailleurs, il n'en va pas ainsi dans les pays où l'industrialisation est récente et les syndicats inexpérimentés. Comment alors aider ces syndicats à atteindre un degré d'évolution analogue à celui des syndicats existant de longue date? Ils ne peuvent pas se permettre de se développer aussi lentement que les grands mouvements syndicalistes du XIXe siècle. Les rapporteurs suggèrent alors une aide technique, une assistance qui peut leur venir soit des syndicats plus anciens, des organisations internationales des travailleurs et de l'Organisation internationale du travail elle-même.

Une conclusion particulière se rapportant à l'utilisation des fonds pour Pac- tion politique est très intéressante: « Lorsque les syndicats, conformément à la décision de leurs membres, consacrent une partie de leurs fonds à des activités politiques, les statuts devraient disposer que ces fonds seront versés à un compte spécial, distinct des comptes généraux du syndicat, et que les membres qui ne désirent pas contribuer au fonds politique peuvent s'abstenir de le faire tout en restant membres de plein droit et sans être désavantagés de ce fait $»$.

Quant à la question si délicate de la surveillance de la gestion financière par les pouvoirs publics, les conclusions sont très sages et très prudentes: \&La surveillance exercée sur l'administration des fonds généraux du syndicat par les pouvoirs publics ne devrait pas dépasser normalement le cadre des mesures nécessaires pour contrôler si le syndicat respecte les dispositions législatives expresses, par exemple s'il présente des relevés financiers annuels, et elle ne devrait en aucun cas porter atteinte au droit que l'on reconnaît généralement aux syndicats d'établir leurs statuts et leurs règlements intérieurs, d'élire leurs représentants en toute liberté et d'organiser la gestion de leur activité... Lorsque des sanctions peuvent être infligées à un syndicat qui utilise ses ressources à des fins illicites ou contrevient d'une autre façon aux dispositions législatives relatives à la gestion des biens syndicaux, ces sanctions, à l'exception de la révocation de l'enregistrement, devraient être infligées exćlusivement par les tribunaux, en conformité de la procédure judiciaire régulière, et non par la voie administrative, les syndicats devraient avoir le droit d'interjeter appel devant les tribunaux de la révocation de l'enregistrement $\$$.

Alors qu'en certains milieux, on exerce des pressions sur l'Etat pour accroître les mesures législatives de façon à règlementer davantage l'action syndicale, avant de faire quoi que ce soit, il serait très utile de connaître l'expérience des autres pays contenue dans cet ouvrage et les conclusions des rapporteurs.

\section{GÉRARD Dion}

The Japanese Factory: Aspects of It: Social Organization. By James C. Abegglen. Glencoe, Ill.: The Free Press, 1958.155 pp. $\$ 3.50$. 
Voici un petit ouvrage sur certains aspects de la réalité industrielle japonaise qui invite les Occidentaux que nous sommes à une réexamen du concept d'efficacité dans l'industrie.

Le développement industriel phénoménal du Japon, qui dépasse de beaucoup en rapidité et en ampleur celui des autres pays asiatiques, pose un gros point d'interrogation aux industriels et aux théoriciens (sociologues et économistes en particulier). Pourquoi, en somme, les Japonais ont-ils si bien assimilé et adapté à leurs fins la technologie industrielle de l'Occident, alors que tant d'autres nations africaines ou asiatiques éprouvent des difficultés culturelles si grandes à absorber le processus de l'industrialisation?

D’après les économistes classiques et les théoriciens-sociologiques comme Marshall, Pareto, Sombart, Weber, Toennies et combien d'autres, la société industrielle moderne se caractérise avant tout par la rationnalité des relations économiques en général, de l'organisation des entreprises et des techniques de production. Puisque donc tout et tous dans l'industrie sont si raisonnables, il devrait être facile de «motiver» les travailleurs économiquement; car, en bonne logique, ces derniers sont libres de poursuivre leurs intérêts économiques « rationnellement », d'acheter biens et services à meilleur marché, de se déplacer professionnellement et géographiquement, de signer les contrats les plus avantageux; de même, la direction sera libre d'embaucher ou de congédier son personnel au gré de ses besoins «raisonnables». On connaît d'ailleurs la thèse de Weber sur la bureaucratie, assise sur la compétence technique, illustrée par la hiérarchisation des positions, impersonnelle et efficace.

Or, la réalité japonaise que nous découvre Abegglen s'accommode fort mal de ces schèmes théoriques. L'auteur observe de près plus de cinquante entreprises industrielles nippones. Il y fait, en gros, les «découvertes» suivantes: 1) l'entreprise japonaise se sent responsable de son personnel bien au delà des strictes relations de production; 2) la rémunération du personnel n'est que partiellement monétaire; elle se fonde sur des critères sociaux généraux plutôt que sur des critères de production: l'âge, l'instruction, l'ancienneté et le nombre d'enfants prennent souvent le pas sur des considérations économiques de rendement; 3) la tâche d'un individu a un véritable caractère de permanence; au Japon, on trouve peu de congédiements, de mises à pied, de mobilité géographique; 4) la structure formelle de l'entreprise est très élaborée; les décisions s'y prennent collectivement, et les responsabilités sont collectives plutôt qu'individuelles; 5) la position (《status 》) à l'usine est le prolongement de la position sociale globale; et 6 ) le recrutement s'appuie moins sur les exigences objectives de la tâche que sur certaines qualités personnelles d'instruction, de caractère et d'arrière-plan social ou économique.

C'est dire que le Japon s'est donné une structure industrielle adaptée à ses valeurs sociales, et notamment familiales. De sorte que ce que le système perd en rationnalité, en flexibilité et en « efficacité », il le gagne en stabilité, en solidarité, en loyauté et en adaptation plus facile au mode de vie industriel.

Le livre est fort bien écrit et très instructif par les comparaisons qu'il suggere et par l'obligation où il place les Occidentaux de reconnaître la relativité de leurs éléments culturels et de leurs thèses sur les impératifs de l'organisation et du développement industriels.

\section{Roger Chartier}

Longer Life. By George H. Soule. New York: The Viking Press, 1958. $151 \mathrm{pp}$. $\$ 3.00$.

Voici un tout petit livre qui résume admirablement bien, dans une langue claire et simple, les connaissances acquises jusqu'ici en matière de gérontologie sociale et économique. Rien, en fait, qui fasse avancer la science du vieillissement des humains, mais beaucoup pour la vulgariser.

L'auteur aligne les causes les plus courantes de la mort, définit expérimentalement le vieillissement, souligne les aptitudes relatives ( au travail et ailleurs) des personnes qui gagnent en maturité, leurs difficultés économiques, leurs tribulations comme personnes à charge, la ségrégation dont ils sont l'objet et la nécessité sociale de leur réintégration.

En bon économiste, l'auteur aborde assez longuement les aspects économi- 